\title{
Trends in Spinal Pain Management Injections in Academic Radiology Departments
}

\author{
J.J. Freeman, R.K. Kilani, C.D. Lascola, L. Gray, and D.S. Enterline
}

\section{ABSTRACT}

BACKGROUND AND PURPOSE: There is a paucity of information present in the current literature with regard to the role of SPMI performance in academic radiology centers. Our aim was to evaluate the current practice patterns for the performance of SPMIs in academic radiology departments.

MATERIALS AND METHODS: A survey of 186 academic radiology departments in the United States was conducted between March 2009 and May 2009. The survey included questions on departmental demographics, recent trends in departmental SPMI performance, type of physicians who refer to radiology for SPMI performance, types of SPMIs offered, the fraction of total institutional SPMI volume performed by radiologists, and the current state of resident and fellow SPMI training proficiency.

RESULTS: Forty-five of the 186 (21.4\%) surveys were completed and returned. Twenty-eight of the 45 responding departments stated that they performed SPMIs; the other 17 stated that they did not. Among the 28 responding departments that perform SPMIs, 6 (21.4\%), 5 (17.9\%), and $8(28.6 \%)$ stated that the number of departmental SPMIs had, respectively, increased, decreased, or remained stable during the past 5 years. SPMI referrals to radiology were made by orthopedic surgeons, neurologic surgeons, neurologists, psychiatrists, anesthesiologists, and internal medicine physicians. CESIs, SNRBs, facet injections, and synovial cyst aspirations are the most frequently performed injections. Fellows and residents become proficient in $88.5 \%$ and $51.9 \%$, respectively, of SPMI-performing departments. Most departments perform $<50 \%$ of the SPMI volume of their respective institutions.

CONCLUSIONS: Most responding academic radiology departments perform SPMIs. Most fellows and just more than half of residents at SPMI-performing departments achieve SPMI proficiency. For the most part, the number of SPMIs performed in responding departments has been stable during the past 5 years.

ABBREVIATIONS: SPMI = spinal pain management injection; $\mathrm{CESI}=$ central epidural steroid injection; $\mathrm{SE}=$ standard error; SNRB = selective nerve root block

$\mathbf{R}$ ecent data suggest that the use of all treatment modalities for acute and chronic back pain is increasing. ${ }^{1,2}$ Payers and patients alike are increasingly interested in pursuing minimally invasive therapies before authorizing expensive and inconsistently effective surgery options. SPMIs are frequently used as a nonoperative treatment for acute spinal-origin pain and chronic degenerative spinal symptoms in patients for whom noninvasive conservative therapies have failed. Many patients who are not considered viable surgical candi-

\footnotetext{
Received December 26, 2011; accepted after revision March 31, 2012.

From Summit Radiology (J.J.F.), Fort Wayne, Indiana; and Department of Radiology (R.K.K., C.D.L., L.G., D.S.E.), Duke University Medical Center, Durham, North Carolina.

Paper previously presented at: 47th Annual Meeting of the American Society of Neuroradiology, May 16-21, 2009; Vancouver, British Columbia, Canada.

Please address correspondence to Jeffrey J. Freeman, MD, Summit Radiology, 6119 West Jefferson Blvd, Fort Wayne IN 46804-3072; e-mail: Jefreeman1977@ yahoo.com

http://dx.doi.org/10.3174/ajnr.A3167
}

dates or who have not had success with prior surgical procedures also rely on injections for pain relief and improved functionality.

Despite the fact that many radiologists have the technical expertise and medical knowledge to effectively perform SPMIs, not all academic radiology groups have established a spinal pain management practice. Reasons for this phenomenon may include competition from other specialties, local political health care realities, and lack of interest.

Without aggressive advertising and with established competition from other service providers within our institution and region, our pain management case volume has continued to rise during the past 5 years (Fig 1). In the midst of this unanticipated growth, we began to consider whether our experience was generalizable to comparable radiologic settings. It was unclear whether this upward trend in referrals was a localized epiphenomenon or evidence of a larger nationwide trend of academic radiology departments performing an increasing percentage of total institutional SPMI volume. 


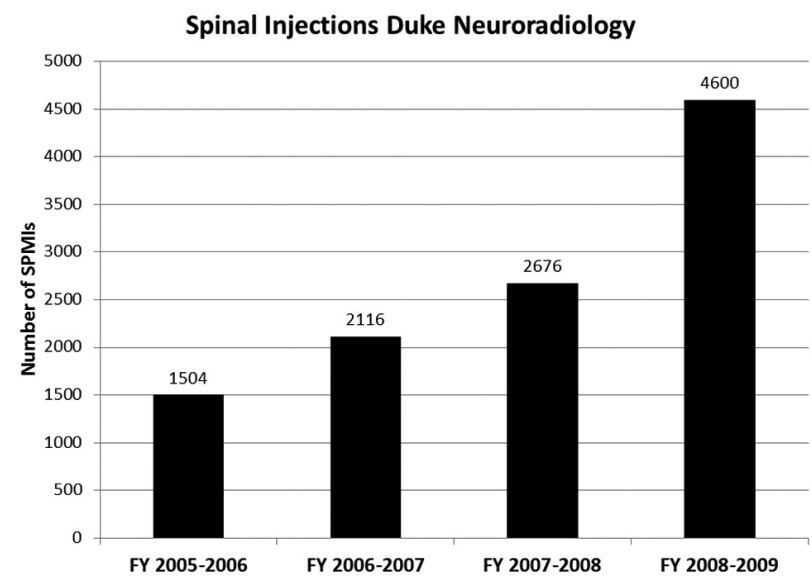

FIG 1. Annual volumes of spinal pain management injections performed by the Duke Neuroradiology Section, 2005-2009.

Furthermore, the role of pain management in radiology training is also a topic of interest. Neuroradiology is the only subspecialty within radiology that includes SPMIs in the fellowshiptraining guideline requirements of the Accreditation Council for Graduate Medical Education. ${ }^{3}$ We wanted to further understand what portion of academic radiology departments include a component of SPMI performance as a part of fellow and/or resident training.

With the medical literature offering no relevant data to characterize recent trends in SPMI volumes; the status of radiology trainee SPMI instruction; and, to a greater extent, the current status of any academic radiology department SPMI parameter, we decided to conduct an SPMI survey of all academic radiology centers in the United States.

This study focuses on discerning the percentage of academic radiology departments with SPMI participation and the nature of the radiology SPMI practice, identifying trends in referral volumes during the past 5 years, and assessing resident and fellow SPMI proficiency in the SPMI-performing departments.

\section{MATERIALS AND METHODS}

A brief survey of the 186 other academic radiology departments in the United States ${ }^{4}$ was conducted between March 2009 and May 2009. Each survey was addressed to the neuroradiology section head. In departments without an identifiable neuroradiology section head, the survey was addressed to the residency director. A request was made to forward the survey to the radiologist in the department in charge of or most experienced with SPMIs in the event that the initial recipient was unable to answer the survey questions. The survey was sent in both hard copy and electronic formats (Survey Monkey, Portland, Oregon). The electronic form of the survey was sent on 3 separate occasions. All recipients were informed of the purpose of the study.

\section{Respondent Demographics}

The recipients were initially asked whether they performed SPMIs, which sections performed SPMIs in their department, and the total number of staff radiologists in their department.

\section{Trends}

Recipients were asked to report the total number of injections performed in their department in the past calendar year and to state their opinion as to whether the total number of SPMIs performed in their department was increasing, decreasing, or stable during the past 5 years.

\section{Referrals}

Recipients were asked which types of physicians make referrals to their department for SPMI performance: anesthesia, psychiatry, neurologic surgery, orthopedic surgery, neurology, or other (a blank was provided to record other specialties).

\section{Procedures Offered}

Recipients were asked whether they offered each of the following procedures: CESI, SNRB, facet injection, synovial cyst aspiration, celiac plexus ablation, or sacroiliac joint injection.

\section{Resident and Fellow Training}

Recipients were asked whether the residents and/or fellows generally became proficient at SPMI performance.

\section{Fraction of Total Institutional Volume}

Recipients were asked to characterize the percentage of the total institutional SPMI procedure volume performed by their department by using the following ranges: $<10 \%, 10 \%-25 \%, 26 \%-$ $50 \%, 51 \%-75 \%$, or $>75 \%$.

\section{RESULTS}

Forty-five of the 186 (21.4\%) distributed surveys were completed and returned. No paper survey forms or e-mailed surveys were returned as undeliverable. Twenty-eight of the 45 (62.2\%) responding departments stated that their department performed SPMIs. The other 17 (37.8\%) respondents stated that their department did not perform SPMIs.

\section{Respondent Demographics}

The mean number of staff radiologists in the departments that perform SPMIs was 87 (range, $8-153$ ). In the departments that did not perform SPMIs, this value was 32 (range, 16-45).

In the 28 departments that perform SPMIs, $23(82.1 \%)$ respondents reported that SPMIs were performed exclusively by members of the neuroradiology section, 4 (14.3\%) reported performance by musculoskeletal section personnel only, and 1 (3.6\%) reported that SPMIs were performed by members of both the neuroradiology and musculoskeletal sections. No responding departments reported that vascular/interventional section members performed SPMIs at their institutions.

In the departments that do not perform SPMIs, the survey was answered by neuroradiology section members in 12 of 17 instances $(70.6 \%)$. For the remaining responses, 1 response was received from members of the vascular/interventional, neurointerventional, general radiology, and body sections. One responding department did not provide a response to this question.

\section{Trends}

In the departments performing SPMIs, the mean annual number of SPMIs performed was $575 \pm 119.3$. 
5-year Trends for Responding Departments

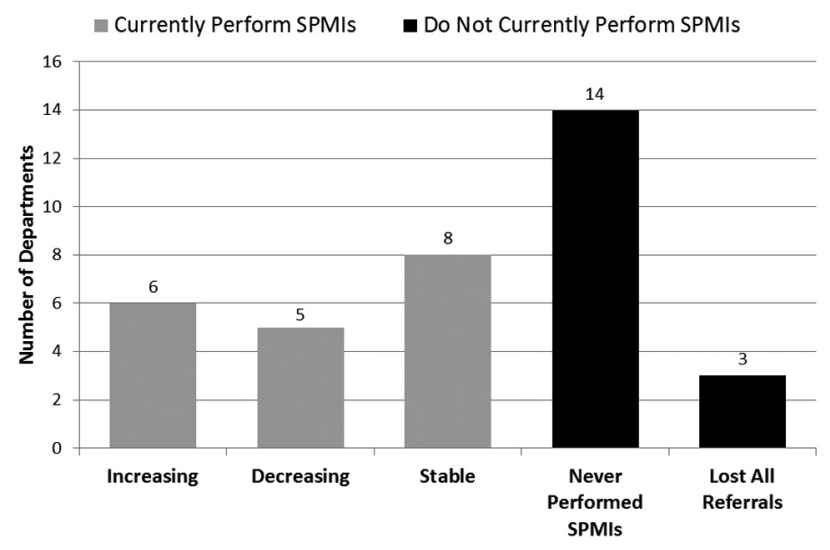

FIG 2. Five-year trends for responding departments from responding departments.

Six of the 28 (21.4\%) departments that perform SPMIs stated that the volume performed by their department has been increasing during the past 5 years. The number of SPMIs performed was decreasing in 5 of the $28(17.9 \%)$ departments. Eight of the 28 (28.6\%) departments stated that the number of yearly SPMIs that they perform has been stable. Nine of the 28 (31.4\%) departments did not answer this question. Fourteen of the 17 (62.4\%) departments not currently performing SPMIs stated that they have never performed SPMIs. Three of the 17 (17.6\%) departments that currently do not perform SPMIs stated that during the past 5 years, they lost all of their referrals and thus stopped performing SPMIs (Fig 2).

\section{Referrals}

Twenty-four of 27 (SE, 5.6) reporting departments stated that they receive referrals from neurologic surgeons. Orthopedic surgeons were reported to have referred patients for SPMIs in 26 of the 27 (SE: 3.4) responding departments. Eighteen of the 27 (SE: 8.4) departments received referrals from neurologists. Physiatrists were reported to have referred to 14 of the 27 (SE, 8.9) responding departments. Eight of the 27 (SE, 8.1) responding departments receive anesthesia SPMI referrals. In addition, 9 of the 27 (SE, 8.4) departments receive referrals from other physicians (internal medicine was indicated once). One responding department did not answer this question.

\section{Procedures Offered}

In the departments that perform SPMIs, 23 of the 28 (SE, 6.7) departments perform CESIs. SNRBs are performed in 25 of the 28 (SE, 5.4) responding departments. Twenty-seven of 28 (SE, 3.2) respondents stated that their departments perform facet injections. Synovial cyst aspirations are performed at 22 of the 28 (SE, 7.2) responding departments, while 4 of the 28 (SE, 6.1) perform sacroiliac joint injections. Celiac plexus ablations are performed at 3 of the 28 (SE, 5.4) responding departments.

\section{Resident and Fellow Training}

In 14 of 27 (51.9\%) responding departments, residents become proficient in SPMI performance. The other 13 (48.1\%) departments think that their residents do not become proficient at SPMI

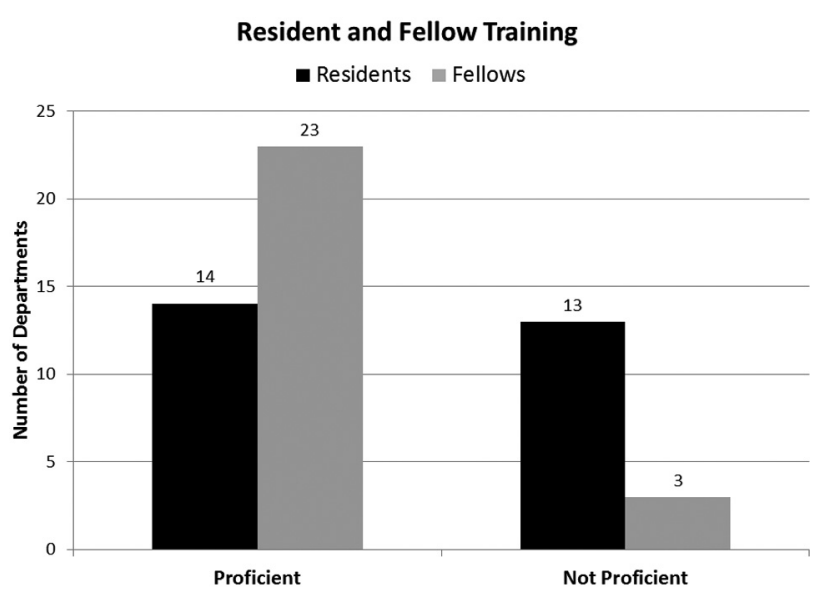

FIG 3. Resident and fellow training proficiency from responding departments.

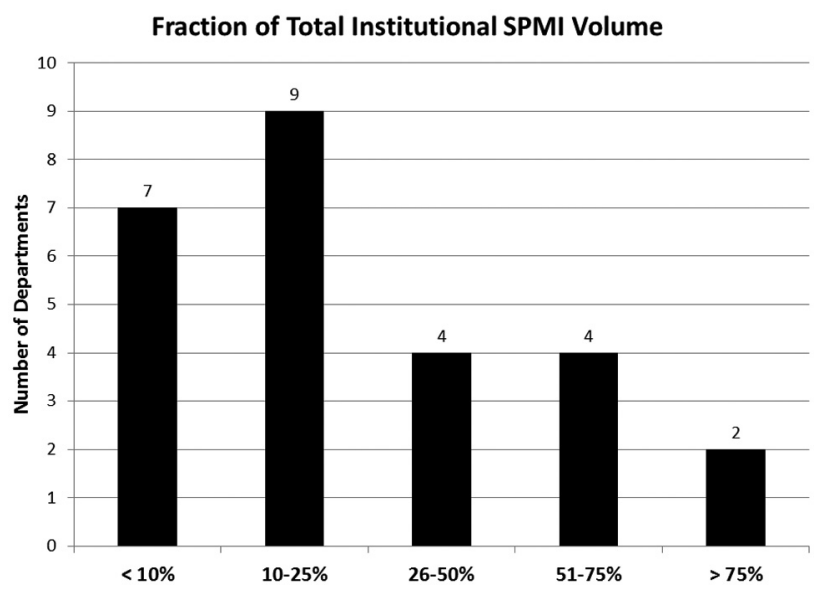

FIG 4. Fraction of total institutional SPMI volume from responding departments.

performance. In 23 of 26 (88.5\%) responding departments with fellows who perform SPMIs, neuroradiology and/or musculoskeletal fellows were reported to achieve proficiency. Only 3 of 26 (11.5\%) reporting departments thought that their fellows did not achieve SPMI proficiency (Fig 3). On the basis of these data, fellows are more likely to become proficient at SPMI performance in comparison with residents $\left(\chi^{2}=6.78 ; P=.004\right)$. One responding department did not answer this question.

\section{Fraction of Total Institutional Volume}

Seven of 26 (SE, 8.1) respondents stated that their department performed $<10 \%$ of the total SPMI volume of their institution. Nine of 26 (SE, 8.7) departments reported that they perform between $10 \%$ and $25 \%$ of their total institutional SPMI volume. Twenty-six percent to $50 \%$ of the total institutional SPMI volume is performed in 4 of 26 (SE, 15.4) responding departments. Four of 26 (SE, 15.4) responding departments performed between $51 \%$ and $75 \%$ of the total SPMI volume of their institution. Two of 26 (SE, 7.7) respondents stated that they perform $>75 \%$ of the total SPMI volume of their institution. Two of the responding departments did not respond to this question (Fig 4). 


\section{DISCUSSION}

SPMIs are performed by nearly two-thirds (63.2\%) of the responding academic departments. In general, these departments have a larger total number of departmental staff radiologists compared with the $36.8 \%$ of responding departments that do not perform SPMIs, 87 and 42 staff radiologists, respectively.

In $82.1 \%$ of responding departments, the neuroradiology section performs the SPMIs exclusively, with musculoskeletal radiologists performing SPMIs exclusively at $14.3 \%$ of the responding departments. A survey in 2000 of neuroradiology fellowships ${ }^{5}$ reported neuroradiology sections performing nonvascular spinal interventions alone in $42 \%$ of cases and sharing the procedures with musculoskeletal sections in $40 \%$. Our results would suggest that neuroradiology sections are performing an increasing relative percentage of SPMIs within radiology departments compared with the year 2000 study. Alternatively, this apparent change may be related to selection bias inherent to the survey.

The range of SPMIs being performed annually in each department is broad, possibly reflecting considerable variability in referral and practice patterns.

Most SPMI-performing radiology departments execute less than half of their total institutional volume, with just under onethird of departments performing between $51 \%$ and $75 \%$ of their total institutional volume. Only 2 departments perform more than $75 \%$ of their institutional total volume. This demonstrates that while radiologists are not the leaders in SPMI performance at most centers, radiology has a conspicuous presence in this performance.

More than 20\% (21.4\%) of radiology departments that perform SPMIs noted an increase in the number of SPMI referrals during the past 5 years; a slightly lower percentage (17.9\%) reported a decrease in the number of referrals, with most $(28.6 \%)$ departments remaining relatively stable. Furthermore, almost one-third $(31.4 \%)$ of the respondents did not answer the question, which most likely reflects uncertainty with regard to the trends within their own departments. Only 3 of the $45(6.7 \%)$ responding departments said that they had completely lost SPMI referrals in the past 5 years. Overall, these results reveal no dominant trend during the past 5 years with regard to SPMI referrals to academic radiology departments.

Inquiries as to the specific reasons why referrals increased, decreased, or were lost at a particular institution were omitted for the sake of maintaining a relatively brief survey and thereby maximizing potential respondent survey participation. The lack of supporting data to explain the reported referral volume trends is certainly a limitation of this survey. Our future intentions to generate follow-up detailed trend-specific surveys to the centers that have gained, lost, or maintained referrals will be the most useful in elaborating the specific factors that are actually affecting SPMI referrals in the responding radiology departments.

Potential factors that could negatively influence the volume of radiology SPMI referrals include competition from other more aggressive SPMI-performing specialties, substandard patient care and patient pain-reduction outcomes, poor radiologist-clinician relations, failure to participate in interdisciplinary conferences, failure to circumvent historic referral patterns, and decreasing and/or lack of radiologist interest in SPMI performance. As the interpretative responsibilities associated with increasing diagnostic imaging volumes climb in academic centers, less radiologist time may be available to devote to performing SPMI procedures. In addition, because cost constraints are increasing in health care, academic institutions may decide that multiple, seemly redundant SPMI-offering services may not be the most cost-effective strategy. In this scenario, administrators may grant SPMI responsibilities exclusively to one department as opposed to several competing stakeholders. Finally, departments that fail to advertise the availability and benefit of their services through passive marketing, diagnostic imaging report recommendations, and direct communications to all potential referrers, from primary care physicians and gerontologists to spine surgeons within their institution and to outside community medical practices, will be at a disadvantage.

To maintain and/or increase SPMI referrals, in our experience, it is paramount for motivated radiology departments to understand and then satisfy the unique needs of both patients and clinicians for a particular institution. Skillful intradepartmental political maneuvering, congenial intradepartmental relations, and favorable patient pain-reduction outcomes are important but may not alone ensure adequate referrals. Radiology departments must surpass expectations to sustain an adequate referral base. For instance, at our institution, commitment to accepting appropriate patient management responsibility and maximizing the patient's preprocedural and postprocedural experience, and not just a favorable intraprocedural patient experience and pain reduction, in our opinion, are the key reasons for the current positive trend of our section in increasing SPMI referrals. Our section performs SPMIs 5 days a week to ensure short patient wait times. One team (staff attending, physician assistant, and fellow) from the neuroradiology section is assigned exclusively to SPMI performance each standard workday. This practice allows sufficient time for our interventionalists to review and discuss, with the patient, pertinent clinical and imaging data; to correlate imaging findings with the distribution and nature of the patient's clinical symptoms (to ensure that the SPMI requested is the most appropriate); and to discuss the pertinent details of the impending procedure and the potential benefit that the patient may expect. This extra attention alleviates patient confusion and fosters reasonable patient expectations.

Patients are directed postprocedurally to contact our section directly for any complications or other issues. Furthermore, we instruct the patient that if a favorable response is achieved and a subsequent SPMI is desired, he or she should contact our section directly. We assume the burden of coordinating with the initial referring physician to determine the appropriateness of further SPMIs in each patient's care plan. Because most of our referrals are from neurosurgery and orthopedic surgery, many of these physicians are happy to relinquish these "nonoperative" patients to our care for long-term SPMI therapy as appropriate. This strategy has generated, for our section, a relatively informed and content cadre of loyal patients, many whom require additional future SPMIs and who elect to have subsequent injections in our department. These patients also relay their favorable experiences to the referring physicians who reward our service with additional referrals. 
At least two-thirds of the responding departments reported receiving SPMI referrals from orthopedic surgeons, neurologic surgeons, and neurologists. Physiatrists made referrals in just more than half of the reporting departments, and anesthesiologists, in under one-third. This is not surprising because anesthesia and physiatry departments are historically more likely to have pain management sections, which readily perform SPMIs, and thereby compete directly with radiology departments for SPMI referrals. This reality is certainly present at our institution. We think that our previously outlined strategy allows us to compete favorably with the other SPMI-performing disciplines at our institution.

CESIs, SNRBs, facet injections, and synovial cyst aspirations are the most frequently performed injections. Each of these injections is performed in $>75 \%$ of the responding departments. Celiac ablations and sacroiliac joint injections are performed at less than a quarter of the responding departments.

Just more than half of the residents (51.9\%) at most SPMIperforming training programs became proficient in SPMI techniques, whereas fellows become proficient $>85 \%$ of the time. This tendency reinforces the notion that fellows generally are more likely to receive procedural training over residents. Fellows, who are closer to the end of their training, will be more aggressive in mastering procedures. Attending physicians will also tend to confer to fellows more responsibility. Residents, initially in the preparation for call and later in their training cycle in preparation for board certification, may tend to sacrifice learning procedures to hone their diagnostic interpretative skills. This is especially true in departments that do not make procedural teaching a priority for residents. Furthermore, to increase the presence of radiology in SPMI performance and increase radiologist SPMI proficiency, motivated institutions may consider initiating radiology pain management fellowships. This may ultimately culminate in radiologist-directed pain management clinics.

Our study has several limitations; the most obvious is the low response rate. Selection bias with regard to radiology subspecialty is another confounding aspect because neuroradiology divisions were the target of the initial survey contact and represent the most frequent performers of SPMIs in these survey data. Further selection bias is possible in that some departments may have chosen not to respond to the survey. Furthermore, the opinion of 1 respondent does not necessarily convey the true nature of the entire SPMI practice at a given institution. Finally, this study did not include data from nonacademic radiology departments and, as a result, may not reflect the specialty-wide trend in pain management within radiology as a whole. A future survey of both academic and community practices and/or analysis of payment data, ideally sponsored and administered by a national society or organization, would be useful in attempting to further elucidate the role of pain management in radiology.

\section{CONCLUSIONS}

Among the responding departments, most perform some SPMIs, though the range of annual volumes is quite broad, and most perform less than half of their total institutional SPMI volume. Neuroradiologists are the most frequent SPMI proceduralists. The most frequently performed procedures are CESIs, SNRBs, facet injections, and synovial cyst aspirations. Most referrals come from orthopedic surgeons, neurologic surgeons, and neurologists. No discernible trend (increasing, decreasing, or stable) was seen across academic radiology departments with regard to the volume of SPMIs being performed during the past 5 years.

\section{REFERENCES}

1. Hart LG, Deyo RA, Cherkin, DC. Physician office visits for low back pain: frequency, clinical evaluation, and treatment patterns from a U.S. national survey. Spine 1995:20:11-19

2. Deyo RA, Mirza SK, Martin BI. Back pain prevalence and visit rates: estimates from U.S. national surveys, 2002. Spine (Phila $\mathrm{Pa}$ 1976) 2006;31:2724-27

3. Accreditation Council for Graduate Medical Education. http://www. ama-assn.org/. Accessed June 8, 2012

4. American Medical Association. http://www.acgme.org/adspublic/ list. Accessed June 8, 2012

5. Friedman DP, Pragmatic BK. Fellowship and practice trends in neuroradiology training programs in the United States. AJNR Am J Neuroradiol 2001;22:1650-53 